\title{
Casimir Effect in Yang-Mills Theory in $D=2+1$
}

\author{
M. N. Chernodub, ${ }^{1,2}$ V. A. Goy, ${ }^{2}$ A. V. Molochkov, ${ }^{2}$ and Ha Huu Nguyen ${ }^{1,3}$ \\ ${ }^{1}$ Institut Denis Poisson UMR 7013, Université de Tours, Tours 37200, France \\ ${ }^{2}$ Laboratory of Physics of Living Matter, Far Eastern Federal University, Sukhanova 8, Vladivostok 690950, Russia \\ ${ }^{3}$ Faculty of Pedagogy, University of Dalat, Lam Dong 671460, Vietnam
}

(Received 4 June 2018; published 6 November 2018)

\begin{abstract}
We study, for the first time, the Casimir effect in non-Abelian gauge theory using first-principles numerical simulations. Working in two spatial dimensions at zero temperature, we find that closely spaced perfect chromoelectric conductors attract each other with a small anomalous scaling dimension. At large separation between the conductors, the attraction is exponentially suppressed by a new massive quantity, the Casimir mass, which is surprisingly different from the lowest glueball mass. The apparent emergence of the new massive scale may be a result of the backreaction of the vacuum to the presence of the plates as sufficiently close chromoelectric conductors induce, in a space between them, a smooth crossover transition to a color deconfinement phase.
\end{abstract}

DOI: 10.1103/PhysRevLett.121.191601

Quantum fluctuations of virtual particles are affected by the presence of physical objects. This property is a cornerstone of the Casimir effect [1], which states that the energy of vacuum ("zero-point") quantum fluctuations should be modified by the presence of physical bodies $[2,3]$. The energy shift of the virtual particles has real physical consequences because the Casimir effect leads to the appearance of a small quantum force, known as the "Casimir-Polder force" [4], between neutral objects. This force has been detected in various experiments [5].

Apart from the simplest cases, the Casimir-Polder forces are difficult to calculate analytically even in noninteracting field theories since the energy spectrum of vacuum fluctuations cannot be determined exactly for generic geometries. Therefore the Casimir effect is often studied using certain analytical approximations such as proximity-force calculations [6] and various numerical tools [7], which include worldline approaches [8] and methods of lattice field theories $[9,10]$.

In interacting theories the calculations become even more involved. In quantum electrodynamics a negligibly small correction to the Casimir-Polder force coming from fermionic vacuum loops was calculated in a second-order perturbation theory $[3,11]$. Perturbative calculations in finite-volume geometries of non-Abelian gauge theories and their effective infrared models were addressed in Ref. [12].

Published by the American Physical Society under the terms of the Creative Commons Attribution 4.0 International license. Further distribution of this work must maintain attribution to the author(s) and the published article's title, journal citation, and DOI. Funded by SCOAP ${ }^{3}$.
In strongly coupled theories the interactions may not only lead to a noticeable modification of the CasimirPolder forces but may also affect the structure of the vacuum itself. The Casimir effect leads to the strengthening of a chiral finite-temperature phase transition in a fourfermion effective field theory [13]. The presence of the boundaries effectively restores the chiral symmetry in an otherwise chirally broken phase both in plane [14] and in cylindrical [15] geometries. The interactions may even change the overall sign of the Casimir-Polder force in certain fermionic systems with condensates [16] and in the $\mathbb{C} P^{N-1}$ model on an interval [17,18]. First-principles numerical simulations show that the presence of the boundaries affects also nonperturbative (de)confining properties of certain bosonic gauge systems [10]. There is evidence that spatially compactified geometries may lead to a phase transition in the non-Abelian vacuum [19].

In our Letter we initiate a first-principles investigation of the Casimir effect in Yang-Mills theory which has an inherently nonperturbative vacuum structure. We consider a zero-temperature Yang-Mills theory in $(2+1)$ spacetime dimensions which exhibits both mass gap generation and color confinement similar to its $(3+1)$-dimensional counterpart. We concentrate on the simplest geometry of two parallel static wires along the $x_{2}$ direction separated by a finite distance $R$ along the $x_{1}$ axis.

The simplest version of the Casimir effect in a gauge system can be formulated in the Maxwellian gauge theory

$$
\mathcal{L}_{U(1)}=-\frac{1}{4} f_{\mu \nu} f^{\mu \nu}, \quad f_{\mu \nu}=\partial_{\mu} a_{\nu}-\partial_{\nu} a_{\mu}
$$

where $a_{\mu}$ is an Abelian gauge field. Restricting ourselves to idealized cases, one may impose either boundary 
conditions corresponding to a material made of a perfect electric conductor (with normal magnetic and tangential electric components vanishing at conductor's boundary) or its dual analogue, an ideal magnetic conductor (in which magnetic and electric components exchange their roles). The electric-type boundary conditions in two spatial dimensions are given by the following condition:

$$
\epsilon^{\mu \alpha \beta} n_{\mu}(x) f_{\alpha \beta}(x)=0,
$$

where $n_{\mu}(x)$ is a vector normal to the boundary at the point $x$. In the geometry of two parallel wires the vacuum fluctuations of the $U(1)$ gauge field lead to the attractive potential between the wires, $V_{\mathrm{Cas}}(R)=-\zeta(3) /\left(16 \pi R^{2}\right)$, where $\zeta(x)$ is the zeta function $[10,20]$.

The Lagrangian of Yang-Mills theory has the form

$$
\mathcal{L}_{\mathrm{YM}}=-\frac{1}{4} F_{\mu \nu}^{a} F^{\mu \nu, a},
$$

where $F_{\mu \nu}^{a}=\partial_{\mu} A_{\nu}^{a}-\partial_{\nu} A_{\mu}^{a}+g f^{a b c} A_{\mu}^{b} A_{\nu}^{c}$ is the fieldstrength tensor of the non-Abelian (gluon) field $A_{\mu}^{a}$ with $a=1, \ldots, N_{c}^{2}-1$, and $f^{a b c}$ are the structure constants of the $S U\left(N_{c}\right)$ gauge group. A non-Abelian analogue of the perfect conductor condition (2) is straightforward as given by the following gauge-invariant condition:

$$
\epsilon^{\mu \alpha \beta} n_{\mu}(x) F_{\alpha \beta}^{a}(x)=0, \quad a=1, \ldots, N_{c}^{2}-1 .
$$

The perfectly conducting chromoelectric wires are positioned at points $x_{1}=0, R$ so that $n_{\mu}=\delta_{\mu 1}$.

In a tree order one may formally set $g=0$ so that both the Yang-Mills theory (3) and the boundary conditions (4) are reduced to $N_{c}^{2}-1$ noninteracting copies of the Maxwell electrodynamics (1) with the $U(1)$ boundary conditions (2). Thus in a tree order all $N_{c}^{2}-1$ gluons contribute additively to the Casimir energy density:

$$
V_{\text {Cas }}^{\mathrm{tree}}=-\left(N_{c}^{2}-1\right) \frac{\zeta(3)}{16 \pi R^{2}} .
$$

The lattice version of the $N_{c}=2$ Yang-Mills theory (3) is given in terms of the $S U(2)$ link variables $U_{l}$ residing on the links $l \equiv l_{x, \mu}$ of the Euclidean cubic lattice $L_{s}^{3}$ with periodic boundary conditions in all three directions. The path integral is given by the integration with the Haar measure over all link variables $U_{l}$ and the standard plaquette action $S_{P}=\beta_{P}\left(1-\frac{1}{2} \operatorname{Tr} U_{P}\right)$. Here the plaquette field strength is $U_{P_{x, \mu \nu}}=U_{x, \mu} U_{x+\hat{\mu}, \nu} U_{x+\hat{\nu}, \mu}^{\dagger} U_{x, \nu}^{\dagger}$, where $\hat{\mu}$ is a unit lattice vector in the positive $\mu$ direction. In the absence of the Casimir wires the lattice couplings $\beta_{P}$ are uniform, $\beta_{P}=\beta$, where the bulk coupling constant,

$$
\beta=\frac{4}{a g^{2}},
$$

is related to the lattice spacing $a$. The quantity $g^{2}$, which has the dimension of mass, becomes the physical coupling of the continuum Yang-Mills theory in the limit $a \rightarrow 0$.

The lattice analogue of the chromoelectric boundaries (4) is realized via the space-dependent coupling [10]: $\beta_{P}=$ $\lambda_{w} \beta$ at the plaquettes $P=P_{x, 23}$ with $x_{1}=0, R$, and $\beta_{P}=\beta$ otherwise. In the limit $\lambda_{w} \rightarrow+\infty$ the tangential chromoelectric field vanishes $\left(U_{P_{23}} \rightarrow \mathbb{1}\right)$, leading to the perfect "chromometallic" conditions (4).

The energy of the vacuum fluctuations of the gluon field is related to a local expectation value of its energy density,

$$
T^{00}=\frac{1}{2}\left(\boldsymbol{B}_{z}^{2}+\boldsymbol{E}_{x}^{2}+\boldsymbol{E}_{y}^{2}\right)
$$

which is a component of the energy-momentum tensor associated with the Yang-Mills Lagrangian (3). In a Minkowski spacetime one has $F_{01}^{a}=E_{x}^{a}, F_{02}^{a}=E_{y}^{a}$, and $F_{12}^{a}=-B_{z}^{a}$, with $a=1,2,3$ and $\boldsymbol{E}_{x}^{2} \equiv\left(E_{x}^{a}\right)^{2}$, etc.

After a Wick rotation to a Euclidean space the energy density (7) transforms to $T_{E}^{00}=\left(\boldsymbol{B}_{z}^{2}-\boldsymbol{E}_{x}^{2}-\boldsymbol{E}_{y}^{2}\right) / 2$, while the geometry of the problem implies that $\left\langle\boldsymbol{B}_{z}^{2}\right\rangle=\left\langle\boldsymbol{E}_{x}^{2}\right\rangle$. Thus in Euclidean space the expectation value of the normalized energy density (7) becomes as follows:

$$
\mathcal{E}_{R}(x)=\frac{1}{2}\left(\left\langle\boldsymbol{E}_{y}^{2}\right\rangle_{0}-\left\langle\boldsymbol{E}_{y}^{2}(x)\right\rangle_{R}\right),
$$

where the subscripts 0 and $R$ indicate that the expectation value is taken, respectively, in the absence of the wires and in the presence of the wires separated by the distance $R$. The ultraviolet divergencies cancel in Eq. (8) so that $\mathcal{E}_{R}(x)$ provides us with a local finite quantity, the Casimir energy density, which is equal to a change in the energy density of the vacuum fluctuations due to the presence of the wires.

In the lattice notations the Casimir energy density (8) calculated per a unit length of the wires is given by

$$
V_{\text {Cas }}^{\text {lat }}(R)=-\left\langle\left\langle S_{P_{23}}\right\rangle\right\rangle_{R}^{\text {lat }},
$$

where the plaquette $P_{23}$ is oriented along the wires' direction $(\mu=2)$ and the Euclidean time $(\nu=3)$, and

$$
\langle\langle\mathrm{O}(x)\rangle\rangle_{R}^{\text {lat }}=\sum_{x_{1}=0}^{L_{s}-1}\left[\left\langle\mathrm{O}\left(x_{1}\right)\right\rangle_{R}-\langle\mathrm{O}\rangle_{0}\right] .
$$

In physical units the Casimir potential is

$$
V_{\text {Cas }}(R) \equiv V_{\text {Cas }}^{\text {phys }}(R)=a^{-2} V_{\text {Cas }}^{\text {lat }}(R / a),
$$

where $a$ is the lattice spacing in physical units and $R=a R_{\text {lat }}$. Ideally, the continuum physics $a \rightarrow \infty$ is 
reached as $\beta \rightarrow \infty$ according to Eq. (6), while in practice one deals with finite values of the lattice coupling $\beta$ which affect the extrapolation to continuum with $O\left(a^{n}\right)$ corrections. We estimate that finite-size effects may lead to numerically significant, $10 \%-15 \%$, corrections to the nonAbelian Casimir energy.

We improve the continuum scaling at finite $\beta$ in three steps. First, in order to reduce the finite-size corrections, we use the mean-field improved coupling expressed via the average plaquette following Refs. [21,22]:

$$
\beta_{\mathrm{I}}(\beta)=\beta \frac{1}{2}\left\langle\operatorname{Tr} U_{P}\right\rangle(\beta) .
$$

Second, we express the physical lattice spacing $a$ via the phenomenologically determined series over $1 / \beta_{I}$ [21],

$$
a \sqrt{\sigma}=\frac{1.341(7)}{\beta_{I}}-\frac{0.421(51)}{\beta_{I}^{2}}+O\left(1 / \beta_{I}^{3}\right),
$$

where $\sigma$ is the tension of the confining (fundamental) YangMills string at zero temperature. In the selected range of the coupling constant $\beta$ the higher-order terms in Eq. (13) are numerically irrelevant.

Third, we notice that in the lattice perturbation theory the expectation value the lattice plaquette operator $\left\langle\operatorname{Tr} U_{\mu \nu}\right\rangle$ acquires radiative corrections, of both an additive and a multiplicative nature. The additive corrections-which correspond to the UV-divergent perturbative vacuum contributions-are automatically removed from the Casimir energy by the subtraction scheme (10). The multiplicative correction originates from the fact that the physically relevant quantity is the product $\beta^{4}\left\langle\operatorname{Tr} U_{\mu \nu}\right\rangle \sim a^{-4}\left\langle\operatorname{Tr} U_{\mu \nu}\right\rangle \sim$ $\left\langle F_{\mu \nu}^{2}\right\rangle_{\text {phys }}$ and not the expectation value of the plaquette itself (indeed, it is the former quantity that determines the physical value of the nonperturbative gluon condensate [23]). In order to improve the finite-size scaling, we thus rescale the expectation value of the plaquette operator with the improved value of the coupling (12): $\left\langle\operatorname{Tr} U_{\mu \nu}\right\rangle \rightarrow\left\langle\operatorname{Tr} U_{\mu \nu}\right\rangle_{I}=\left(\beta_{I} / \beta\right)^{4}\left\langle\operatorname{Tr} U_{\mu \nu}\right\rangle$.

Summarizing, the scale-improved relation for the Casimir energy density in the continuum limit is

$$
V_{\mathrm{Cas}}(R)=-\frac{1}{a^{2}(\sigma, \beta)}\left(\frac{\beta_{I}}{\beta}\right)^{4}\left\langle\left\langle S_{P_{23}}\right\rangle\right\rangle_{R}^{\mathrm{lat}},
$$

where the lattice spacing $a=a(\sigma, \beta)$ and the mean-field improved lattice coupling $\beta_{I}$ are given in Eqs. (13) and (12), respectively. In the weak coupling limit $\beta \rightarrow \infty$ the energy (14) approaches its natural form given by Eqs. (9) and (11) since the plaquette expectation value then tends to unity $\frac{1}{2}\left\langle\operatorname{Tr} U_{P}\right\rangle(\beta) \rightarrow 1$, and the improved coupling converges to the Wilson coupling, $\beta_{I}(\beta) \rightarrow \beta$.

We generate gauge-field configurations on the $32^{3}$ lattice using a hybrid Monte Carlo algorithm which combines standard Monte Carlo methods [24] with the molecular dynamics approach, similar to our studies of the Casimir forces in Abelian gauge theories in Refs. [10,25]. Long autocorrelation lengths in Markov chains are eliminated by over-relaxation steps using five steps between trajectories for 250000 trajectories in total.

A non-Abelian permittivity of the wires is determined by the strength of the coupling constant $\lambda_{w} \beta$ at their world surfaces. At large $\lambda_{w}$ the wires behave as almost-ideal conductors which force all tangent components of the chromoelectric field to vanish at the wires, $F_{\|}^{a} \rightarrow 0$.

In Fig. 1 we show the non-Abelian Casimir energy (14) as the function of the interwire distance $R$ in units of the physical string tension $\sigma$ for nearly perfect chromometallic wires with $\lambda_{w}=50$. In order to demonstrate the absence of substantial finite-volume corrections, we also show the results for a larger $L=48$ lattice. The Casimir energy exhibits nearly excellent physical scaling as the data at different values of the bulk lattice coupling $a=a(\beta)$ and at different volumes match the same single curve. We get similar results for other strengths $\lambda_{w}$.

We fit the Casimir energy by the following function:

$$
V_{\mathrm{Cas}}(R)=3 \frac{\zeta(3)}{16 \pi} \frac{1}{R^{2}} \frac{1}{(\sqrt{\sigma} R)^{\nu}} e^{-M_{\mathrm{Cas}} R},
$$

where $\nu$ and $M_{C}$ are the free parameters determined from the best fit. The power of $\sigma$ in the denominator in Eq. (15) is chosen to keep the correct dimension $\left(\right.$ mass $\left.^{2}\right)$ of the Casimir potential, as it corresponds to the Casimir energy of the non-Abelian fluctuations between the wires calculated per unit length of the wire.

The fitting function (15) has a transparent physical meaning. The exponent $\nu$ in the fitting function (15) is an anomalous dimension of the Casimir potential at short distances. The quantity $M_{\text {Cas }}$, which we call the "Casimir mass," corresponds to an effective screening of the Casimir potential at large distances due to nonperturbative mass gap generation. In the absence of interactions the mass gap is

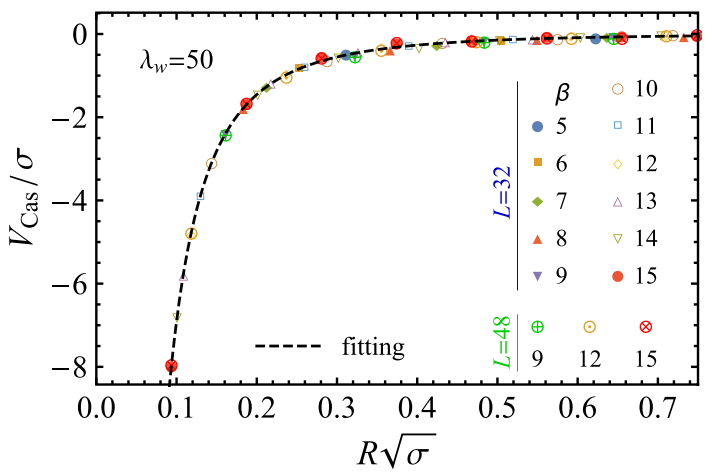

FIG. 1. The Casimir potential $V_{\text {Cas }}$ for a nearly perfect wire $\left(\lambda_{w}=50\right)$ as the function of the distance $R$ between the wires in units of the string tension $\sigma$ at various bulk couplings $\beta$. 
absent, $M_{\text {Cas }}=0$, while the anomalous dimension is equal to its canonical value, $\nu=0$, so the phenomenological potential (15) naturally reduces to its tree-level expression (5).

The best fit of the Casimir potential with the almostperfect wires $\left(\lambda_{w}=50\right)$ is shown in Fig. 1 by the dashed line. The dependences of the best-fit values of the anomalous dimension $\nu$ and the Casimir mass $M_{\text {Cas }}$ on the strength of the wire $\lambda_{w}$ are shown in Fig. 2. They can be described by the fit function

$$
\mathrm{O}\left(\lambda_{w}\right)=\mathrm{O}^{\infty}+\alpha_{\mathrm{O}} e^{-\lambda_{w} / \lambda_{w}^{\circ}}
$$

where $\mathrm{O}=\nu, M_{\text {Cas }}$, and $\mathrm{O}^{\infty}, \alpha_{\mathrm{O}}$ and $\lambda_{w}^{\mathrm{O}}$ are the fitting parameters. The quantities $\nu$ and $M_{\mathrm{Cas}}$ rapidly approach, with $\lambda_{w}^{\nu} \simeq \lambda_{w}^{M_{\text {Cas }}}=12(1)$, and the corresponding asymptotic values $\mathrm{O}^{\infty} \equiv \lim _{\lambda_{w} \rightarrow \infty} \mathrm{O}\left(\lambda_{w}\right)$ in the perfect-wire limit.

The asymptotic value of the Casimir mass $M_{\text {Cas }}$ corresponding to the energy of the vacuum fluctuations of the non-Abelian gauge field between perfect wires $\left(\lambda_{w} \rightarrow \infty\right)$,

$$
M_{\mathrm{Cas}}=1.38(3) \sqrt{\sigma}
$$

is shown by the horizontal dashed line in the main part of Fig. 2. Surprisingly, the Casimir mass $M_{\text {Cas }}$, Eq. (17), turns out to be substantially smaller than the mass

$$
M_{0^{++}} \approx 4.7 \sqrt{\sigma}
$$

of the lowest $0^{++}$glueball in $S U(2)$ gauge theory (calculated numerically in Refs. [21,26]). According to Fig. 2, the enhancement of the strength of the wires leads to a diminishing of the Casimir mass. On the contrary, as the wire weakens, the Casimir mass moves towards the higher masses, so we may expect that in the weak-wire limit $\lambda_{w} \rightarrow 1$ the Casimir mass $M_{\text {Cas }}$ may naturally approach the mass of the lightest glueball, $M_{0^{++}}$, although the simple

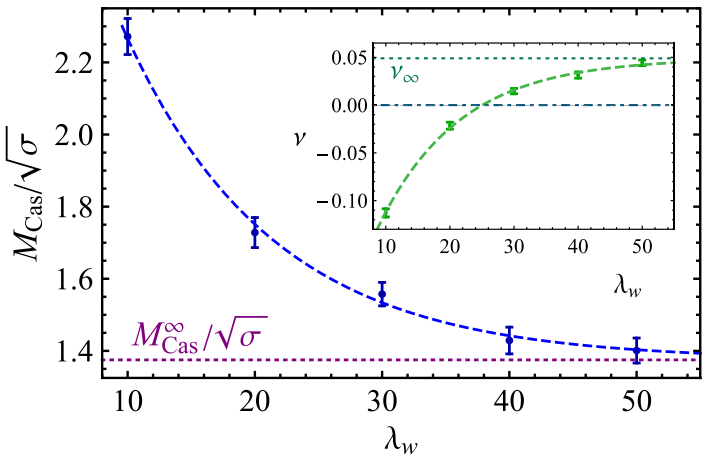

FIG. 2. The Casimir mass $M_{\mathrm{Cas}}$ and the anomalous dimension $\nu$ (in the inset) for the best fit (15) as a function of the strength of the wire $\lambda_{w}$. The thick dashed lines denote the best fit by function (16), and the short-dashed horizontal lines show the asymptotic values (17) and (19), respectively. The dotted-dashed line in the inset marks the tree-level power $\nu=0$. form of the fitting function (16) does not allow us to make this conclusion more precise at the present stage.

According to the fit in the inset of Fig. 2, the anomalous dimension $\nu$ slightly overshoots the free-field value $\nu=0$ :

$$
\nu_{\infty}=0.05(2) .
$$

One may also suggest that in the volume between finitely separated parallel wires the gluons behave as if they are subjected to a heat bath at finite temperature. In a Euclidean formulation of an equilibrium finite-temperature theory, the temporal direction is compactified to a circle with the length $1 / T$, making the fields periodic along this direction. On the contrary, the periodicity of the gluon fields is evidently absent in the Casimir setup. However, similar to the $T \neq 0$ case, the perfectly conducting wires do indeed restrict allowed frequencies of free gluons with certain polarizations. In particular, the propagator of free gluons in the Feynman gauge corresponds to the Neumann boundary condition for the normal (with respect to the boundary) gluon component $A_{\perp}^{a} \equiv A_{1}^{a}$ [27]. For narrowly placed wires the Neumann conditions dimensionally reduce the dynamics of the normal gluon components $A_{\perp}^{a}$ to $(1+1)$ dimensional spacetime with the tangential coordinate $x_{\|}=\left(x_{2}, x_{0} \equiv x_{3}\right)$. Thus the normal gluon component $A_{\perp}^{a}$ in the Casimir setup plays the role of a timelike gluon $A_{0}^{a}$ at $T \neq 0$. Therefore in our case one could expect that the normal gluon components $A_{\perp}^{a}$ are correlated along the conducting wires with the "Casimir" screening mass $M_{g, \text { Cas }}^{2}=c_{D} g^{2} /(2 \pi R)$, where $c_{D}$ is a constant.

Yang-Mills theories are known to experience a deconfinement phase transition at a sufficiently high temperature. In $(2+1)$ dimensions the critical temperature in $S U(2)$ gauge theory was determined in Ref. [28]. Given the mentioned analogy, one may expect the gluonic vacuum in between sufficiently close wires may enter a deconfinementlike regime. A similar conclusion may also be drawn from properties of a confining compact QED in finite geometries [25]. In order to check this idea, we calculate numerically the deconfinement order parameter, the Polyakov line $L$, which has a vanishing expectation value in the confinement phase, $\langle L\rangle=0$, and a nonzero value in the deconfinement phase, $\langle L\rangle \neq 0$.

The Polyakov line is given by an ordered product of the non-Abelian matrices along the temporal direction:

$$
L_{x}=\frac{1}{2} \operatorname{Tr} \prod_{x_{3}=0}^{L-1} U_{x, x_{3} ; 3}
$$

where $\boldsymbol{x} \equiv\left(x_{1}, x_{2}\right)$ is the spatial two-dimensional coordinate. Notice that the Polyakov line (20) is defined along the long temporal direction, while in the finite-temperature theory the line is directed along the short compactified 


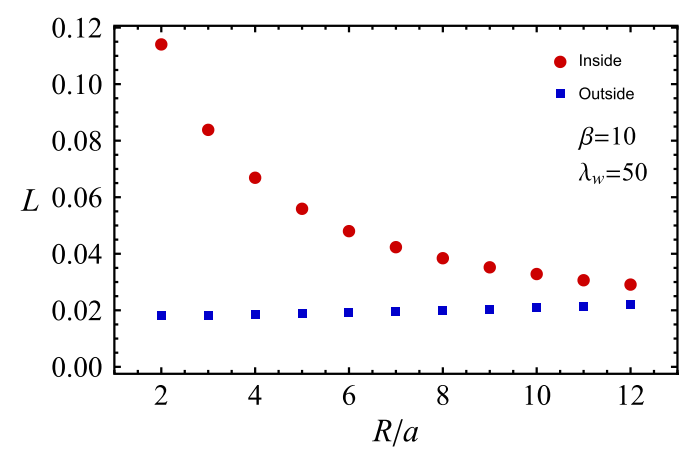

FIG. 3. A typical expectation value of the absolute value of the mean Polyakov line in the spaces in between and outside the wires vs the interwire separation $R$.

time. This property shows a difference between the Casimir-like geometry and a finite-temperature theory.

We calculated the expectation value of the absolute value of a normalized sum over the Polyakov lines (20) over the points in the space inside and outside the wires. For all sets of parameters we get the same picture: as the distance between the wires gets smaller, the expectation value of the Polyakov line in between the wires increases, thus signaling an approach to a deconfinement regime, Fig. 3. However, we have not found any signature of a critical behavior of the Polyakov lines, in agreement with the smooth behavior of the Casimir potential as a function of the interwire distance $R$, Fig. 1. Therefore the gluons in between the wires become deconfined, and the transition in between the confining and deconfining regions is, most probably, a smooth noncritical crossover or an infinite-order transition of a BerezinskiiKosterlitz-Thouless type [29].

Summarizing, we studied for the first time the Casimir effect in a zero-temperature non-Abelian gauge-field theory using first-principles simulations. We found that at large distances between the perfectly chromo-conducting wires the attractive Casimir interaction is an exponentially diminishing function of the interwire separation. The infrared damping of the Casimir interaction is characterized by the new quantity, the Casimir mass (17), which is unexpectedly 3 times lighter than the mass of the lowest glueball (18). As the chromometallic wires become more opaque $\left(\lambda_{w} \rightarrow 1\right)$, the Casimir mass increases, presumably towards the lowest glueball mass.

The short-distance Casimir interaction is slightly different than the canonical tree-level $R^{-2}$ behavior (5) due to a small anomalous scaling dimension (19).

Finally, we observed a (smooth) confinementdeconfinement transition of the gluonic fields in between the wires. The relatively low value of the Casimir mass may be a result of the gradually induced deconfinement in a shrinking finite geometry, which weakens the mass gap generation of the zero-temperature Yang-Mills theory.

The numerical simulations were performed at the computing cluster Vostok-1 of Far Eastern Federal University.
The research was carried out within the state assignment of the Ministry of Science and Education of Russia (Grant No. 3.6261.2017/8.9). We are thankful to D. Karabali and V.P. Nair for letting us know about their work [30] prior to its publication. The work of H. H. N. has been supported by a Ph.D. fellowship of Campus France.

Note added.-Recently, we were informed about Ref. [30], which shows that the $(2+1)$ Casimir mass is related to the magnetic mass of a gluon in $(3+1)$ dimensions.

[1] H. B. G. Casimir, On the attraction between two perfectly conducting plates, Proc. Kon. Ned. Akad. Wetensch. 51, 793 (1948).

[2] M. Bordag, G. L. Klimchitskaya, U. Mohideen, and V. M. Mostepanenko, Advances in the Casimir Effect (Oxford University Press, New York, 2009).

[3] K. A. Milton, The Casimir Effect: Physical Manifestations of Zero-Point Energy (World Scientific Publishing, Singapore, 2001).

[4] H. B. G. Casimir and D. Polder, The Influence of retardation on the London-van der Waals forces, Phys. Rev. 73, 360 (1948).

[5] S. K. Lamoreaux, Demonstration of the Casimir Force in the 0.6 to $6 \mu \mathrm{m}$ Range, Phys. Rev. Lett. 78, 5 (1997); U. Mohideen and A. Roy, Precision Measurement of the Casimir Force from 0.1 to $0.9 \mu \mathrm{m}$, Phys. Rev. Lett. 81, 4549 (1998); G. Bressi, G. Carugno, R. Onofrio, and G. Ruoso, Measurement of the Casimir Force between Parallel Metallic Surfaces, Phys. Rev. Lett. 88, 041804 (2002).

[6] B. V. Derjaguin, I. I. Abrikosova, and E. M. Lifshitz, Direct measurement of molecular attraction between solids separated by a narrow gap, Q. Rev. Chem. Soc. 10, 295 (1956); J. Blocki, J. Randrup, W. J. Swiatecki, and C. F. Tsang, Proximity forces, Ann. Phys. (N.Y.) 105, 427 (1977).

[7] S. G. Johnson, Numerical methods for computing Casimir interactions, Lect. Notes Phys. 834, 175 (2011).

[8] H. Gies and K. Klingmuller, Worldline algorithms for Casimir configurations, Phys. Rev. D 74, 045002 (2006); H. Gies, K. Langfeld, and L. Moyaerts, Casimir effect on the worldline, J. High Energy Phys. 06 (2003) 018.

[9] O. Pavlovsky and M. Ulybyshev, Casimir energy in the compact QED on the lattice, arXiv:0901.1960; Casimir energy in noncompact lattice electrodynamics, Theor. Math. Phys. 164, 1051 (2010); Casimir energy calculations within the formalism of the noncompact lattice QED, Int. J. Mod. Phys. A 25, 2457 (2010); Monte Carlo calculation of the lateral Casimir forces between rectangular gratings within the formalism of lattice quantum field theory, Int. J. Mod. Phys. A 26, 2743 (2011).

[10] M. N. Chernodub, V. A. Goy, and A. V. Molochkov, Casimir effect on the lattice: U(1) gauge theory in two spatial dimensions, Phys. Rev. D 94, 094504 (2016); Nonperturbative Casimir effect and monopoles: Compact Abelian gauge theory in two spatial dimensions, Phys. Rev. D 95, 074511 (2017). 
[11] M. Bordag, D. Robaschik, and E. Wieczorek, Quantum field theoretic treatment of the Casimir effect, Ann. Phys. (N.Y.) 165, 192 (1985).

[12] A. Chodos, R. L. Jaffe, K. Johnson, C. B. Thorn, and V. F. Weisskopf, New extended model of hadrons, Phys. Rev. D 9, 3471 (1974); T. H. Hansson and R. L. Jaffe, Cavity quantum chromodynamics, Phys. Rev. D 28, 882 (1983); T. Hashimoto, QCD Casimir effects with bag boundary at finite temperature, Prog. Theor. Phys. 73, 1223 (1985); A. J. Stoddart and R. D. Viollier, Cavity quantum chromodynamics to order $\alpha_{\mathrm{s}}^{2}$, Phys. Lett. B 208, 65 (1988); G. U. Schreiber and R. D. Viollier, The gluon self-energy in cavity QCD, Ann. Phys. (N.Y.) 215, 277 (1992); F. Canfora and L. Rosa, Casimir energy in the Gribov-Zwanziger approach to QCD, Phys. Rev. D 88, 045025 (2013).

[13] A. Flachi, Strongly Interacting Fermions and Phases of the Casimir Effect, Phys. Rev. Lett. 110, 060401 (2013).

[14] B. C. Tiburzi, Chiral symmetry restoration from a boundary, Phys. Rev. D 88, 034027 (2013).

[15] M. N. Chernodub and S. Gongyo, Interacting fermions in rotation: chiral symmetry restoration, moment of inertia and thermodynamics, J. High Energy Phys. 01 (2017) 136; Effects of rotation and boundaries on chiral symmetry breaking of relativistic fermions, Phys. Rev. D 95, 096006 (2017).

[16] A. Flachi, M. Nitta, S. Takada, and R. Yoshii, Sign Flip in the Casimir Force for Interacting Fermion Systems, Phys. Rev. Lett. 119, 031601 (2017).

[17] A. Flachi, M. Nitta, S. Takada, and R. Yoshii, Casimir Force for the $\mathbb{C} P^{N-1}$ Model, arXiv:1708.08807.

[18] A. Betti, S. Bolognesi, S. B. Gudnason, K. Konishi, and K. Ohashi, Large-N $\mathbb{C} \mathbb{P}^{\mathrm{N}-1}$ sigma model on a finite interval and the renormalized string energy, J. High Energy Phys. 01 (2018) 106.

[19] E. Elizalde, S. D. Odintsov, and A. Romeo, Effective potential for a covariantly constant gauge field in curved space-time, Phys. Rev. D 54, 4152 (1996).
[20] J. Ambjorn and S. Wolfram, Properties of the vacuum. I. Mechanical and thermodynamic, Ann. Phys. (N.Y.) 147, 1 (1983).

[21] M. J. Teper, $\mathrm{SU}(N)$ gauge theories in $(2+1)$ dimensions, Phys. Rev. D 59, 014512 (1998).

[22] G. Parisi, Recent progresses in gauge theories, AIP Conf. Proc. 68, 1531 (1981); B. Lucini and M. Teper, SU(N) gauge theories in $(2+1)$ dimensions: Further results, Phys. Rev. D 66, 097502 (2002).

[23] A. Hietanen, K. Kajantie, M. Laine, K. Rummukainen, and Y. Schroder, Plaquette expectation value and gluon condensate in three dimensions, J. High Energy Phys. 01 (2005) 013.

[24] C. Gattringer and C. B. Lang, Quantum Chromodynamics on the Lattice (Springer-Verlag, Berlin, 2010).

[25] M. N. Chernodub, V. A. Goy, and A. V. Molochkov, Casimir effect and deconfinement phase transition, Phys. Rev. D 96, 094507 (2017).

[26] A. Athenodorou and M. Teper, $\mathrm{SU}(N)$ gauge theories in $2+1$ dimensions: Glueball spectra and k-string tensions, J. High Energy Phys. 02 (2017) 015.

[27] C. Peterson, T. H. Hansson, and K. Johnson, Loop diagrams in boxes, Phys. Rev. D 26, 415 (1982).

[28] M. Teper, The finite temperature phase transition of SU(2) gauge fields in $(2+1)$ dimensions, Phys. Lett. B 313, 417 (1993).

[29] V. L. Berezinskii, Destruction of long-range order in onedimensional and two-dimensional systems having a continuous symmetry group. I. Classical systems, Sov. Phys. JETP 32, 493 (1970); Destruction of long-range order in one-dimensional and two-dimensional systems having a continuous symmetry group. II. Quantum systems, Sov. Phys. JETP 34, 610 (1971); J. M. Kosterlitz and D. J. Thouless, Ordering, metastability and phase transitions in two-dimensional systems, J. Phys. C 6, 1181 (1973).

[30] D. Karabali and V.P. Nair, Casimir effect in $(2+1)$ dimensional Yang-Mills theory as a probe of the magnetic mass, arXiv:1808.07979. 